\title{
NUMERICAL STUDY OF THE THERMALLY CONDUCTIVE FINITE THICKNESS WALLS IMPACT ON HEAT TRANSFER REGIME IN A CLOSED SYSTEM IN CONDITIONS OF RADIANT ENERGY SUPPLY
}

\author{
A. E. Nee ${ }^{1 a}$ \\ 'National Research Tomsk Polytechnic University, 634050 Tomsk, Russia
}

\begin{abstract}
Plane problem of thermogravitational convection in a closed rectangular cavity is numerically solved in conditions of radiant energy supply to the one of the boundaries. Differential heat transfer parameters (fields of temperatures and stream functions) for the conjugate (only vertical walls, only horizontal, vertical and horizontal walls) and the nonconjugate formulation are obtained. Temperature distributions in the $\mathrm{Y}$ direction in the cross section along the axis of symmetry showed that the presence of heat-conducting finite thickness walls leads to a redistribution of the energy which is accumulated by gas and enclosure structures.
\end{abstract}

\section{Introduction}

In order to reduce the energy consumption for heating of the large premises it is advisable to abandon the traditional convective heating in many cases. The most promising is the use of gas infrared emitters for the local heating of the limited size areas where it is needed [1].

An approach [2], which is based on the solution of the heat balance equation, is suggested for the analysis of heating systems with gas and electric infrared emitters. The algorithm [2] allows us to calculate the required number of emitters and their capacity. But the heat transfer model [2], as well as its further modification [3], is formulated without taking into account the free convection movement of air masses and the conjugation of gas and thermally conductive enclosure structures. Investigation of jointly proceeding processes of conduction and convection in a closed system with a source of radiant energy in conjugate formulation is of interest.

The aim of this study is numerical investigation of the thermally conductive finite thickness walls impact on heat transfer regime in a closed system in a closed system with a source of radiant energy.

\section{Problem formulation and solution method}

Solution domain of the boundary value problem of unsteady conductive - convective heat transfer is presented in Figure 1 . Conditions of symmetry were accepted on the external boundaries. Equalities of temperatures and heat flows were set on the interfaces "gas - wall".

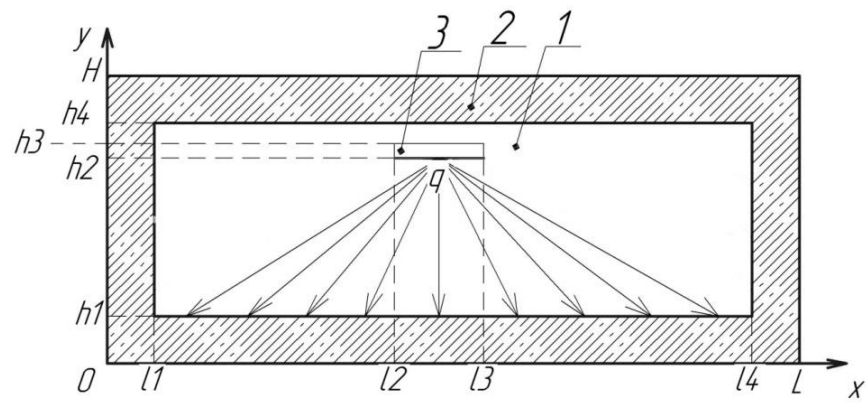

Figure 1. Solution domain: 1 - air; 2 - thermally conductive walls; 3 - gas infrared emitter (symbolic notation)

${ }^{\text {a }}$ Corresponding author : nee_alexander@mail.ru 


\section{MATEC Web of Conferences}

At the problem formulation was assumed that the gas is heat-conducting viscous incompressible Newtonian fluid, satisfying the Boussinesq approximation and absolutely transparent fluid for emission. It was considered that radiation-reflective coatings were applied on the vertical walls. The radiant flux, which was coming from the infrared emitter, was uniformly accumulated only by the lower horizontal interface "gas - wall".

Differential equations of conjugate thermogravitational convection are written in accordance with the theory [4 - 6]. In dimensionless variables "vorticity stream function temperature " are as follows:

$$
\begin{gathered}
\frac{\partial \Omega}{\partial \tau}+\frac{\partial \Psi}{\partial Y} \frac{\partial \Omega}{\partial X}-\frac{\partial \Psi}{\partial X} \frac{\partial \Omega}{\partial Y}=\sqrt{\frac{\operatorname{Pr}}{\operatorname{Ra}}} \cdot\left(\frac{\partial^{2} \Omega}{\partial X^{2}}+\frac{\partial^{2} \Omega}{\partial Y^{2}}\right)+\frac{\partial \Theta_{1}}{\partial X}, \\
\frac{\partial^{2} \Psi}{\partial X^{2}}+\frac{\partial^{2} \Psi}{\partial Y^{2}}=-\Omega, \\
\frac{\partial \Theta_{1}}{\partial \tau}+\frac{\partial \Psi}{\partial Y} \frac{\partial \Theta_{1}}{\partial X}-\frac{\partial \Psi}{\partial X} \frac{\partial \Theta_{1}}{\partial Y}=\frac{1}{\sqrt{\operatorname{Ra} \cdot \operatorname{Pr}}} \cdot\left(\frac{\partial^{2} \Theta_{1}}{\partial X^{2}}+\frac{\partial^{2} \Theta_{1}}{\partial Y^{2}}\right), \\
\frac{\partial \Theta_{2}}{\partial F_{2}}=\frac{\partial^{2} \Theta_{2}}{\partial X^{2}}+\frac{\partial^{2} \Theta_{2}}{\partial Y^{2}} .
\end{gathered}
$$

The initial conditions for the equations (1) - (4) are as follows:

$$
\begin{gathered}
\Psi(X, Y, 0)=0 ; \Omega(X, Y, 0)=0 ; \\
\Theta_{1}(X, Y, 0)=\Theta_{2}(X, Y, 0)=0 .
\end{gathered}
$$

Boundary conditions for equations (1) - (4) are as follows: at the external boundaries of the solution domain:

$$
\frac{\partial \Theta_{2}(X, Y, \tau)}{\partial n}=0
$$

at the interfaces "gas - wall" parallel to the axis X:

$$
\Psi=0, \frac{\partial \Psi}{\partial Y}=0,\left\{\begin{array}{l}
\Theta_{i}=\Theta_{j}, \\
\frac{\partial \Theta_{i}}{\partial Y}=\frac{\lambda_{j}}{\lambda_{i}} \cdot \frac{\partial \Theta_{i}}{\partial Y}+K i,
\end{array} \quad \text { где } \mid \begin{array}{l}
i=\overline{1,2} \\
j=\overline{1,2}
\end{array}\right.
$$

at the interfaces "gas - wall" parallel to the axis Y:

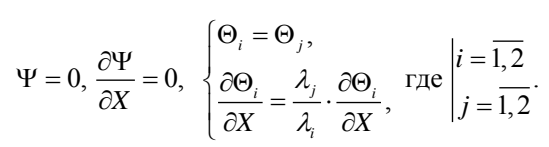

where $F o-$ Fourier number; $R a$ - Rayleigh number; $K i$-Kirpichev number; Pr- Prandtl number; $\lambda-$ coefficient of thermal conductivity, W/(m·K); X, Y - dimensionless coordinates; Indices: 1, 2 - elements of construction (Figure 1).

Formulated boundary problem was solved by finite - difference method on a uniform grid $(111 \times 111)$, analogically [7]. Approximation of equations (1) - (4) made on the basis of a locally one-dimensional A.A. Samarskiy scheme [8]. Obtained one dimensional difference analogues of differential equations were solved by the sweep method [9].

The used algorithm and method of solution was tested on a model problem of thermogravitational convection [10, 11] in a closed square cavity. As the heat transfer characteristics was used an integral criterion (Nusselt number). The test results are shown in Table 1.

Table 1. Comparison of the average Nusselt numbers.

\begin{tabular}{|l|l|l|l|}
\hline Ra & $10^{3}$ & $10^{4}$ & $10^{5}$ \\
\hline This study (grid $81 \times 81)$ & 1,089 & 2,205 & 4,442 \\
\hline$[10]$ & 1,117 & 2,238 & 4,471 \\
\hline$[11]$ & 1,108 & 2,201 & 4,430 \\
\hline
\end{tabular}

Based on the analysis of $N u_{a v}$ values, which are presented in the Table 1, it can be concluded that using algorithm and solution method are able to work. 


\section{Results and discussion}

Numerical study carried out at the following values of dimensionless parameters: $R a=10^{6}, \operatorname{Pr}=0,71, \mathrm{Ki}=1$. The fields of temperatures and stream functions are shown in Figure 2.

Based on the analysis of temperature and stream function fields (Figure 2) it can be concluded that the presence of heatconducting walls of finite thickness leads to significant modifications of the temperature distribution (Fig. 2 a, c, e). Small circulation flows are displaced by large-scale convective cells in the lower corners of the gas cavity (Fig. 2 d, f, h). Heat removal only to vertical enclosure structures (Fig. 2) is reflected in a form of the convective torch. The thickness of the heated gas layer are decreased at the lower horizontal surface in this case. Taking into account only the horizontal heat-conducting walls leads to air velocity reducing (Fig. $2 \mathrm{f}$ ). The value of stream function $(0,004)$ in the center of the vortex (see Fig. $2 \mathrm{f}$ ) is decreased 3 times compared with $\Psi$ for nonconjugate formulation (Fig. 2 b). By analyzing the solutions for different formulations of the
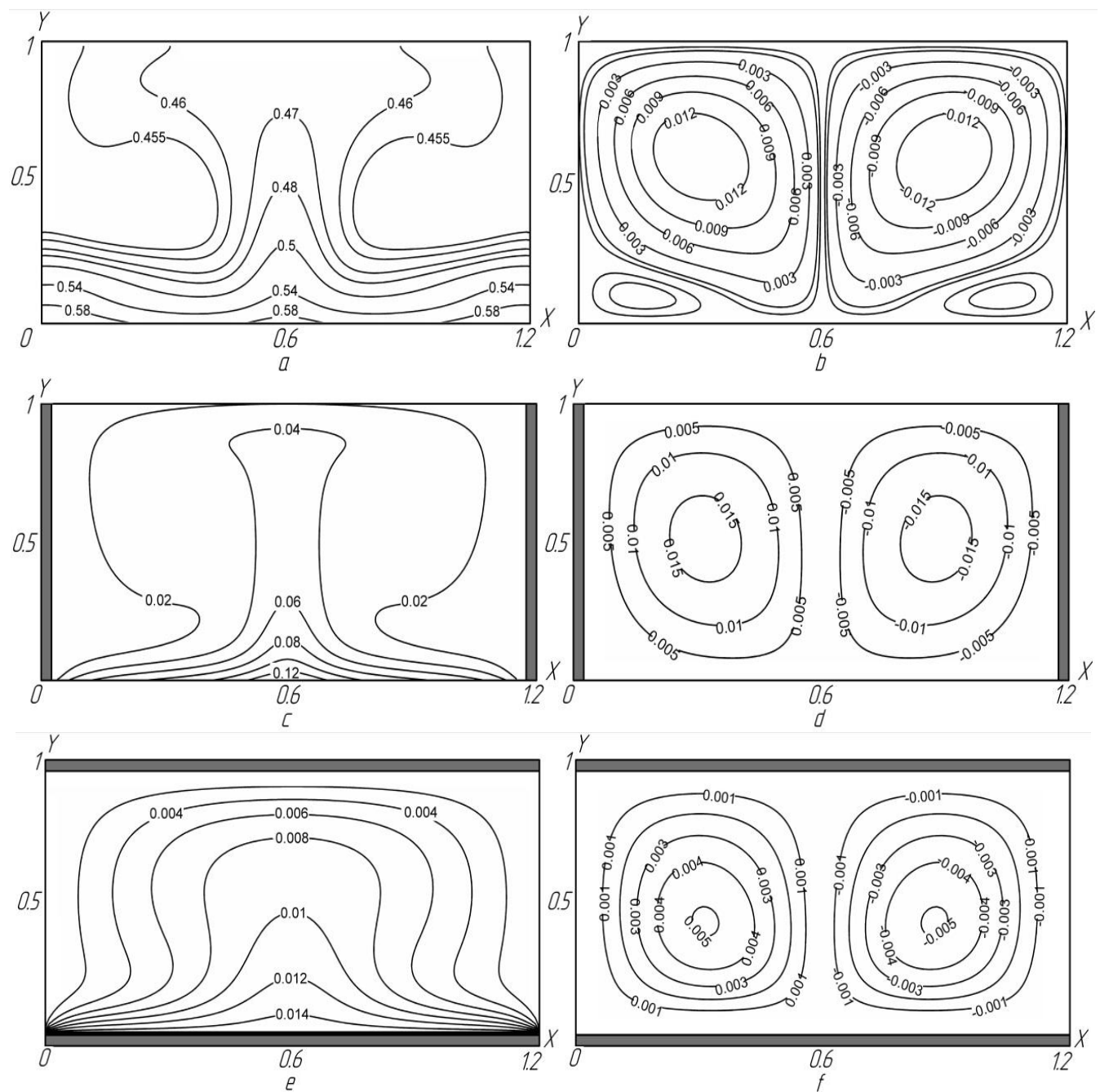


\section{MATEC Web of Conferences}
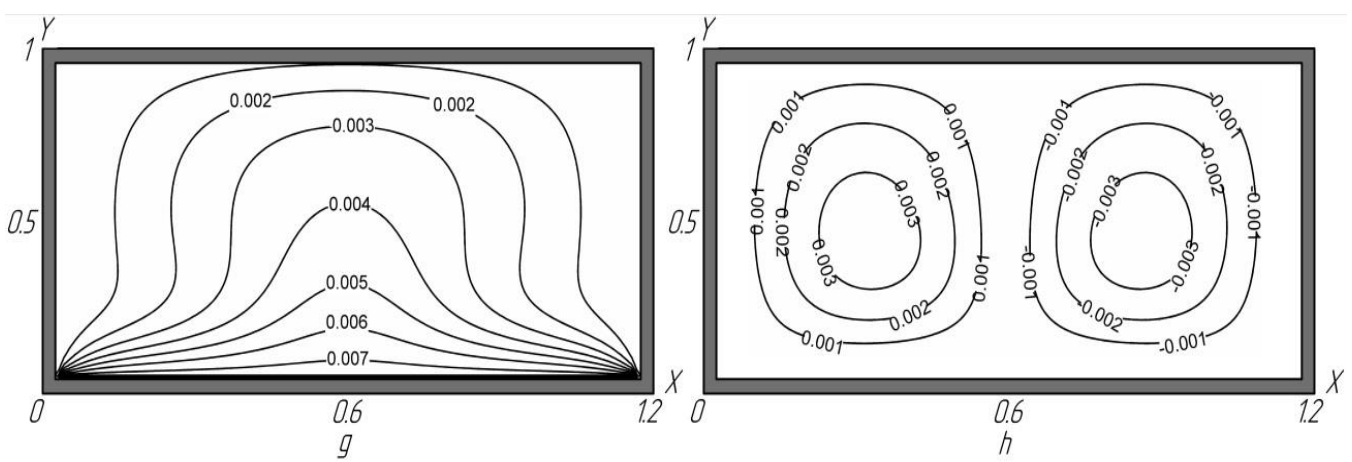

Figure 2. Isolines of temperature (a, c, e, g) and stream function (b, d, f, h) at $\tau=500$.

conductive-convective heat transfer problems it can be concluded that the heat removal to the thermally conductive walls of finite thickness significantly reduces the temperature of the gas cavity. This factor is quite clearly observed on the temperature distribution in the $\mathrm{Y}$ axis direction in the cross section along the axis of symmetry of the solution domain (Fig. 3).

Temperature distributions in the $\mathrm{Y}$ direction in the cross section along the axis of symmetry are presented in figure 3.

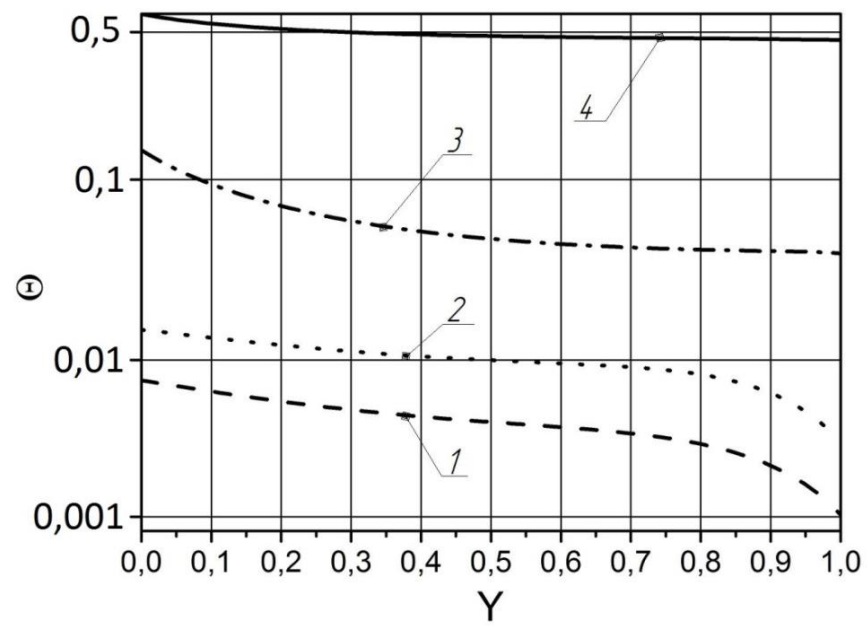

Figure 3. Temperature distributions in the $\mathrm{Y}$ direction in the cross section along the axis of symmetry at $\tau=500: 1-$ heat removal to the vertical and horizontal walls; 2 - heat removal only to the vertical walls; 3 - heat removal only to the horizontal walls; 4 - nonconjugate formulation.

It is clearly seen that the accumulation of heat in the thermally conductive walls of finite thickness leads to a significant decrease in temperature of the gas cavity. This is due to the fact that the part of the thermal energy, which is coming from the infrared emitter, removes to the enclosure structures. Therefore, it is advisable to carry out the investigations of heat transfer in systems with sources of radiant energy in the conjugate formulation.

\section{Conclusion}

According to the results of numerical simulation it was founded that heat removal to the heat-conducting walls of finite thickness leads to significant modifications of differential heat transfer characteristics. The conjugate problem formulation allows to analyses the redistribution of radiant energy between gas and enclosure structures.

The reported investigation was supported by grant of State Task "Nauka” №13.1339.2014/K (code of the Federal Target Scientific and Technical Program no. 2.1410.2014).

\section{References}

1. K. Roth, ASHRAE, 6 (2007)

2. V. V. Buhmirov, S. A. Krupennikov, Yu. S. Solnyshkova, Her. of Ivan. St. Pow. Un. 2 (2009) 


\section{Smart Grids 2015}

3. V. V. Buhmirov, S. A. Krupennikov, Yu. S. Solnyshkova, Her. of Ivan. St. Pow. Un., 4 (2010)

4. G.V Kuznetsov, M.A. Sheremet, T. and A., 16, 1 (2009)

5. Kuznetsov, V.I. Maksimov, T.A. Nagornova, N.I. Kurilenko, G. Ya. Mamontov, J. of Engin. Ph. and Thermoph., 86, 3 (2013)

6. G.V. Kuznetsov, T.A. Nagornova, A.E. Ni, J. of Engin. Ph. and Thermoph., 88, 1 (2015)

7. G.V. Kuznetsov, P.A. Strizhak J. of Engin. Thermoph., 17, 3 (2008)

8. A.A. Samarskii, The Theory of Difference Schemes (Nauka, Moscow, 1977)

9. P. J. Roache, Computational Fluid Dynamics, Hermosa Publishers (Albuquerque, NM, USA, 1976)

10. H.N. Dixit, V. Babu Int. J. H. M. Tr. 49 (2006)

11. H. Sajjadi, R. Kefayatib, Therm. Sc. 19, 1 (2015) 